\title{
Ion-Association Adsorption of Water-Soluble Porphyrin at a Liquid-Liquid Interface and an External Electric Field Effect on the Adsorption
}

\author{
Reiko OKumura, Teruo Hinoue and Hitoshi Watarai ${ }^{\dagger}$ \\ Department of Chemistry, Faculty of Science, Osaka University, \\ Machikaneyama, Toyonaka, Osaka 560, Japan
}

\begin{abstract}
By using total internal-reflection fluorescence (TIRF) spectroscopy it was shown that an unprotonated form of watersoluble porphyrin $(5,10,15,20$-tetraphenyl-21 $H, 23 H$-porphinetetrasulfonic acid, TPPS) is preferentially adsorbed over the diprotonated one by the toluene-water or di(2-ethylhexyl)phthalate (DEHP)-water interface in the presence of a cationic surfactant (cetyltrimethylammonium bromide, CTAB). The pH dependencies of the TIRF intensity were satisfactorily analyzed by a simple adsorption model, including the protonation equilibria in the bulk aqueous phase and the adsorption equilibrium at the interface of TPPS. Further, it was found that applying an electric field across the DEHP-water interface significantly changed the ion-association adsorption equilibria. This result was explained in terms of the electrophoresis of TPPS in the interfacial region.
\end{abstract}

Keywords Liquid-liquid interface, water-soluble porphyrin, ion-association adsorption, total internal fluorescence spectroscopy, electric field effect

In recent years, the adsorption of ionic species at a liquid-liquid interface has become of interest in the fields of solvent extraction, counter-current chromatography and ion-selective liquid-membrane electrodes. Several spectroscopic methods ${ }^{1}$ and an interfacial tension measurement ${ }^{2}$ have been employed for examining the interfacial adsorption behavior of ionic porphyrins and the tris(4,7-diphenyl-1,10-phenanthroline)iron(II) ion. In addition, total internal-reflection fluorescence (TIRF) spectroscopy has also been developed for in situ observations of fluorescent species adsorbed by a liquid-liquid interface. ${ }^{3,4}$

In TIRF spectroscopy ${ }^{5-7}$ the fluorescence comes from the optical excitation of chemical species by an evanescent wave, which develops on the side of an optically rarer phase at the interface when the excitation light is totally reflected from the interface between the optically rarer and denser phases. Because the evanescent wave rapidly decays with increasing distance from the interface, typically within a distance of one wavelength, the fluorescent species existing in the interfacial region are selectively excited. In addition, by adjusting the observation angle from which the fluorescence is observed $^{5,6}$ it is possible to observe the fluorescence coming from a defined interfacial region. Because of such spatial selectivities, TIRF spectroscopy is a useful technique for an interfacial study. In this work, TIRF spectroscopy was applied to determine the chemical form

† To whom correspondence should be addressed. of $5,10,15,20$-tetraphenyl-21 $H, 23 H$-porphinetetrasulfonic acid (TPPS) at liquid-liquid interfaces and to examine the $\mathrm{pH}$ dependence of the interfacial adsorption of TPPS in the presence of the cationic surfactant by the aid of a stoichiometric analysis.

On the other hand, the control of interfacial reactions and the selective concentration of materials by external means, such as an electric field, are interesting subjects in separation chemistry. Although the effects of dc and ac electric fields on the solvent dispersion and phase separation efficiencies have been investigated ${ }^{8}$, they have rarely been discussed in terms of the reaction which takes place at the liquid-liquid interface under an electric field. ${ }^{9}$ We therefore attempted to controll the ionassociation adsorption of TPPS by applying a dc electric field across the interface.

\section{Experimental}

A Hitachi 650-40 fluorescence spectrophotometer was used for fluorescence measurements. Figure 1 illustrates the optical arrangement in a sample compartment of the spectrophotometer. Two right-angle prisms were attached to the cell walls facing the excitation light source and the fluorescence detector, respectively. The excitation light beam passing through the prism and the cell wall was impinged upon the organic solvent-water interface in the cell at an angle of incidence of $\mathrm{ca} .72^{\circ}$, sufficiently greater than the critical angle of $62.9^{\circ}$ for the 


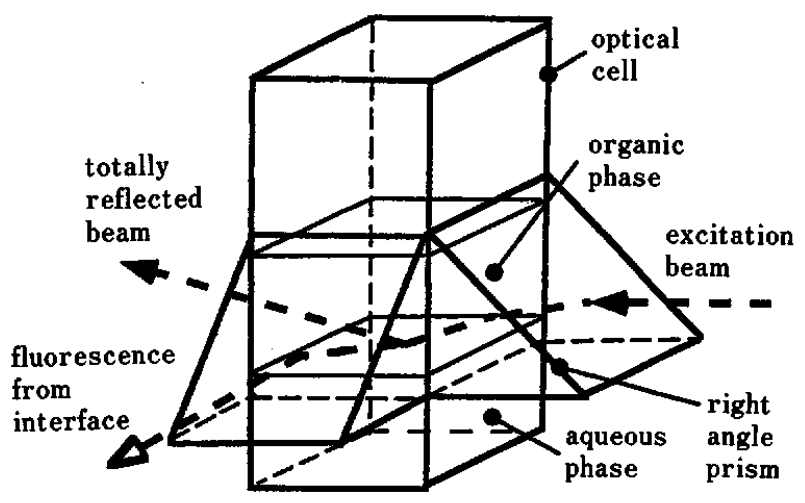

Fig. I Schematic drawing of the optical arrangement for TIRF measurements.

toluene/water system and $63.8^{\circ}$ for the DEHP/water system. The penetration depth ${ }^{10}$ was calculated to be $134 \mathrm{~nm}$ at the toluene-water interface and $142 \mathrm{~nm}$ at the DEHP-water interface for an excitation wavelength of $420 \mathrm{~nm}$. The fluorescence from the interface was taken out through the prism and detected at a right angle from the excitation light beam with an observation angle of $72^{\circ}$, the same as the angle of incidence. The upper inside of the optical quartz cell $(10 \mathrm{~mm})$ was treated with dichlorodimethylsilane in benzene so as to render the cell wall hydrophobic and to prepare a flat interface. All of the measurements were performed at $25 \pm 0.1^{\circ} \mathrm{C}$. The electric field was applied using a high-voltage power supply (Matsusada Precision Devices, HCZE-30P No. 25) between two platinum wire electrodes, partially covered with a Teflon tube and dipped in the organic and aqueous phases. The electrode in the aqueous phase was grounded throughout the experiment.

The absorption spectra of TPPS aqueous solutions were measured with a JASCO Ubest 550 spectrophotometer. From the pH dependence of the spectra, the dissociation or protonation constants of TPPS were determined. The $\mathrm{pH}$ was measured with a Horiba F-14 $\mathrm{pH}$ meter.

The porphyrin used in this work was $5,10,15,20$ tetraphenyl-21 $H, 23 H$-porphinetetrasulfonic acid (TPPS, see Fig. 2), which was purchased from Dojindo and used without further purification.

Cetyltrimethylammonium bromide (CTAB) from Wako Chemicals was used as a cationic surfactant. Toluene and di(2-ethylhexyl)phthalate (DEHP) were used as organic solvents. Toluene (Nakarai G. R.) was fractionally distilled and DEHP (Tokyo Kasei G. R.) was washed with a dilute sodium hydroxide solution and water, then dried by evaporation. The aqueous solutions were prepared with Milli-Q water and their $\mathrm{pHs}$ were adjusted with sodium hydroxide, sodium acetate and perchloric acid solutions so that the ionic strength of the solutions was kept constant at 0.01 . TPPS ( -4 charged) was very soluble in the aqueous phase, and was not extracted into the organic phase in the $\mathrm{pH}$ region examined, even in the presence of the cationic surfactant.

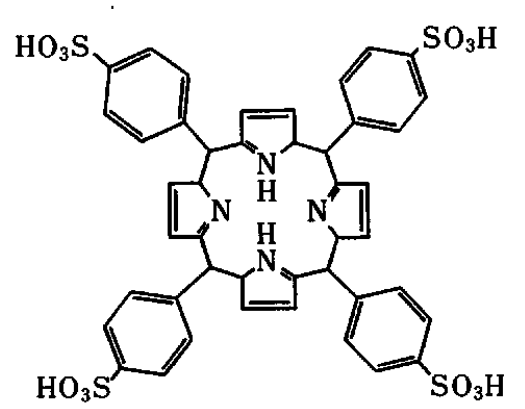

Fig. 2 Molecular structure of TPPS. Protonation occurs on the two nitrogen atoms in the porphyrin ring.

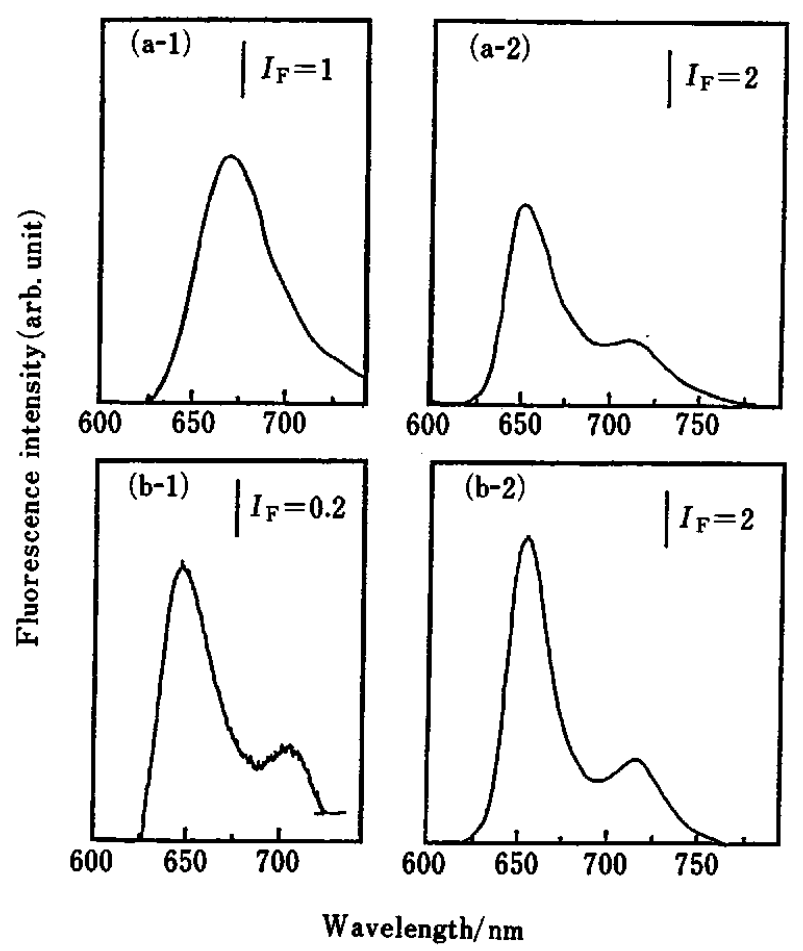

Fig. 3 Aqueous-phase fluorescence spectra and TIRF spectra of TPPS in a toluene-water system. (a-1) aqueous phase spectra, excitation at $436 \mathrm{~nm}$, pH 3.09 ; (a-2) TIRF, $420 \mathrm{~nm}$, pH 3.09; (b-1) aqueous phase spectra, $415 \mathrm{~nm}, \mathrm{pH} 5.64$; (b-2) TIRF, $420 \mathrm{~nm}$, pH 5.64; The aqueous phase contained $0.989 \times 10^{-7} \mathrm{~mol} \mathrm{dm}^{-3}$ TPPS and $0.996 \times 10^{-7} \mathrm{~mol} \mathrm{dm}^{-3} \mathrm{CTAB}$.

\section{Results and Discussion}

Fluorescence spectra of TPPS in the presence of cetyltrimetylammonium bromide (CTAB)

Figure 3 shows the aqueous-phase fluorescence spectra (conventional spectra) and the TIRF spectra of TPPS at the toluene-water interface in the presence of a cationic surfactant, i.e., CTAB. No TIRF spectra were observed in the absence of CTAB. The surfactant cation resides at the interface to form ion-pairs with anionic species in the aqueous phase. The ionassociation equilibria of TPPS with CTAB at the 
interface was attained within $1 \mathrm{~h}$. At $\mathrm{pH} 3.09$, the aqueous-phase spectrum (a-1) showed a single strong band at $670 \mathrm{~nm}$, whereas the TIRF spectrum (a-2) showed a weak band with a shoulder. This difference in shape indicates that TPPS exists in different chemical forms between in the bulk and at the interface. As is known, the diprotonated TPPS successively dissociates with increasing $\mathrm{pH}$, that is, $\mathrm{H}_{2} \mathrm{TPPS}^{2-} \rightleftarrows \mathrm{HTPPS}^{3-}+\mathrm{H}^{+}$ and HTPPS ${ }^{3-} \rightleftarrows$ HTPPS $^{4-}+\mathrm{H}^{+}{ }^{11,12}$ According to the $\mathrm{p} K$ values for these dissociation equilibria, determined to be $4.60 \pm 0.01\left(\mathrm{p} K_{1}\right)$ and $5.17 \pm 0.01\left(\mathrm{p} K_{2}\right)$ spectrophotometrically in this work, it is expected that the diprotonated form is dominant in the bulk at pH 3.09. Consequently, the aqueous-phase spectrum is assigned to the diprotonated form. On the other hand, at $\mathrm{pH} 5.64$, both spectra in the bulk (b-1) and at the interface (b-2) were essentially identical, except for a difference in intensity. This fact indicates that TPPS exists in the same form in the bulk as well as at the interface; it is further suggested from the $\mathrm{p} K$ values that both the aqueous phase and the TIRF spectra are due to the unprotonated TPPS. On the other hand, a similarity between the spectra at $\mathrm{pH} 5.64$ (b-1 and b-2) and the TIRF spectrum at pH 3.09 (a-2) implies that TPPS exists in the unprotonated form at the interface, even though the $\mathrm{pH}$ is 3.09 in the bulk.

The differences in intensity between the aqueous-phase spectra (a-1 and b-1) and between the TIRF spectra (a-2 and b-2) are complementary to each other. Namely, when the $\mathrm{pH}$ is changed from 3.09 to 5.64 , the intensity for the bulk decreases, while that for the interface increases. From this observation and the spectral change discussed above, it is suggested that TPPS in the bulk tends to be concentrated in the unprotonated form onto the interface with increasing pH. Further, because such a $\mathrm{pH}$ dependence of the conventional and TIRF spectra could not be found in the absence of the cationic surfactant, it is concluded that this concentration is caused by ion-association adsorption in which the unprotonated TPPS forms an ion-pair with the cationic surfactant.

As described above, TPPS exists in the unprotonated form at the interface, even at $\mathrm{pH} 3.09$, where the diprotonated form is dominant in the bulk (see a-2). This implies that the ion-pair between TPPS and the surfactant is so stable that TPPS tends not to be protonated. The result from a stoichiometric analysis has indicated that the unprotonated TPPS and the cationic surfactant are ion-associated with a ratio of 1 to 1. In addition, $\mathrm{X}$-ray diffraction analyses have pointed out that the molecular structure of the protonated form exhibits great derivations from planarity of the porphyrin ring. ${ }^{13}$ Such knowledge concerning the structure of the ion-pair implies that the planarity of the porphyrin ring is important concerning the stability of the ion-pair.

To examine the effect of the polarity of the organic phase on ion-association adsorption, the aqueous phase and TIRF spectra were also measured for the DEHP/ water system. These spectra showed a $\mathrm{pH}$ dependence

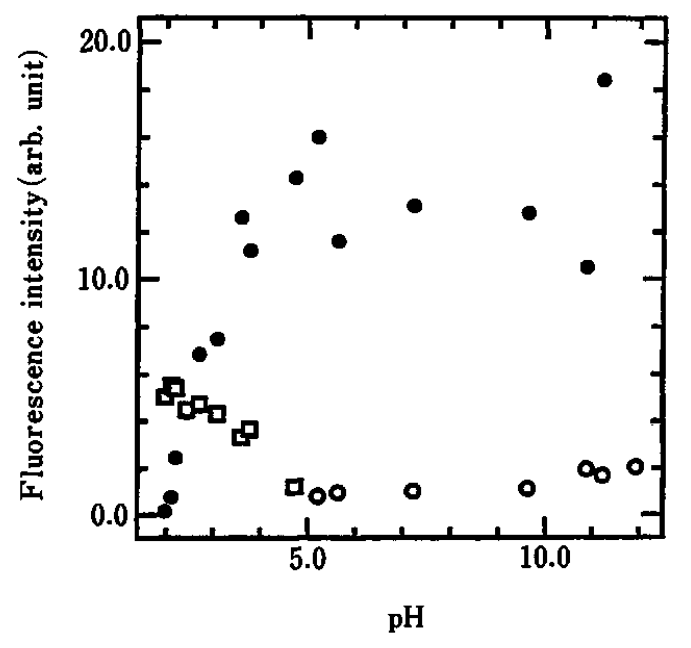

Fig. $4 \mathrm{pH}$ dependencies of the aqueous phase and TIR fluorescence intensities in a toluene-water system. Solid circle, TIRF intensity of the unprotonated TPPS, excitation at $420 \mathrm{~nm}$; open square, aqueous phase fluorescence intensity of the protonated TPPS, $436 \mathrm{~nm}$; open circle, aqueous phase fluorescence intensity of the unprotonated TPPS, $415 \mathrm{~nm}$; All the sample solutions contained $0.989 \times 10^{-7} \mathrm{~mol} \mathrm{dm}^{-3}$ TPPS and $0.996 \times 10^{-7} \mathrm{~mol} \mathrm{dm}^{-3}$ CTAB.

similar to that for the toluene/water system, suggesting that TPPS exists in the unprotonated form at the DEHP-water interface in the presence of the cationic surfactant. However, the adsorption constant at this interface was very different from that at the toluenewater interface.

\section{Adsorption constants at toluene-water and DEHP-water interfaces}

Figure 4 shows the $\mathrm{pH}$ dependence of the fluorescence intensity for the toluene/water system. As can be seen in this figure, the TIRF intensity (solid circle), being proportional to an interfacial concentration of the unprotonated TPPS, increased with $\mathrm{pH}$ in the range from 2 to 6, and leveled off above pH 6. On the other hand, the intensity for the protonated form in the bulk of the aqueous phase (open square) decreased with $\mathrm{pH}$ to 5 , and was not detected above $\mathrm{pH} 5$, as expected from the values of $\mathrm{p} K_{1}$ and $\mathrm{p} K_{2}$. In addition, no significant fluorescence of the unprotonated form in the bulk (open circle) could be observed in the $\mathrm{pH}$ range above 5 where the unprotonated form would be dominant, indicating that most of the unprotonated form was adsorbed by the interface.

Such a pH dependence was stoichiometrically analyzed by considering the dissociation equilibrium in the bulk and assuming that the unprotonated TPPS was all adsorbed by the interface before the adsorption was saturated. The dissociation constant $\left(K_{\mathrm{a}}\right)$ for $\mathrm{H}_{2}$ TPPS $^{2-} \rightleftarrows$ TPPS $^{4-}+2 \mathrm{H}^{+}$is equal to $K_{1} \times K_{2}$, its value was calculated to be $10^{-9.77}$. The apparent ion-association adsorption constant $\left(K_{\mathrm{ad}}\right) \mathrm{TPPS}^{4-} \rightleftarrows \mathrm{TPPS}_{\mathrm{i}}^{4-}$ is expressed by 


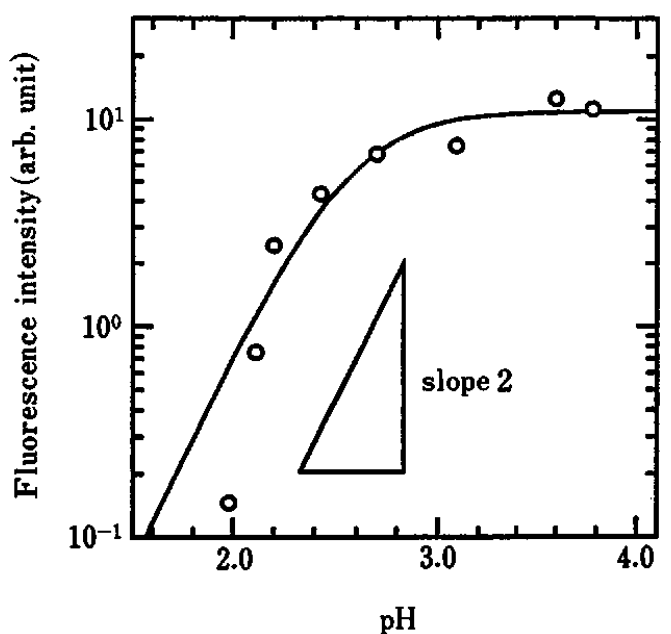

Fig. 5 Best-fitted curve resulting from a stoichiometric analysis of the TIRF intensities with an adsorption constant of $45 \mathrm{~cm}$ at the toluene-water interface.

$$
K_{\mathrm{ad}}=\left[\text { TPPS }^{4-}\right]_{\mathrm{i}} /\left[\text { TPPS }^{4-}\right] \text {, }
$$

where subscript $i$ refers to the interface. Because the TIRF intensity $\left(I_{\mathrm{F}}\right)$ is proportional to the interfacial concentration of the unprotonated form, the intensity is expressed by

$$
I_{\mathrm{F}}=\phi_{\mathrm{i}} \times\left[\mathrm{TPPS}^{4-}\right]_{\mathrm{i}}
$$

Here, $\phi_{\mathrm{i}}$ is a constant which contains the fluorescence efficiency of the unprotonated form and the collection efficiency, depending on the TIRF assembly. Using the relationship [TPPS $]_{\mathrm{t}}=\left[\mathrm{TPPS}^{4-}\right]+\left[\mathrm{H}_{2}\right.$ TPPS $\left.^{2-}\right]+$ $\left[\text { TPPS }^{4-}\right]_{\mathrm{i}} \times\left(S_{\mathrm{i}} / V\right)$, Eq. (2) is rewritten as

$$
I_{\mathrm{F}}=\phi_{\mathrm{i}} K_{\mathrm{ad}} K_{\mathrm{a}}[\mathrm{TPPS}]_{\mathrm{t}} /\left\{K_{\mathrm{a}}\left(1+K_{\mathrm{ad}} S_{\mathrm{i}} / V\right)+\left[\mathrm{H}^{+}\right]^{2}\right\}
$$

where [TPPS $]_{\mathrm{t}}$ is the total concentration of TPPS, and $S_{\mathrm{i}}$ and $V$ denote the interfacial area and the volume of the aqueous phase, respectively. By curve-fitting the TIRF intensities to Eq. (3) in the $\mathrm{pH}$ range from 2 to 4 , the value of $K_{\mathrm{ad}}$ was determined to be $45 \mathrm{~cm}$ for a toluene/water system containing $0.996 \times 10^{-7} \mathrm{~mol} \mathrm{dm}^{-3} \mathrm{CTAB}$. In this fitting, $0.989 \times 10^{-7} \mathrm{~mol} \mathrm{dm}^{-3}$ for [TPPS], $1.0 \mathrm{~cm}^{2}$ for $S_{\mathrm{i}}$, and $1.2 \mathrm{~cm}^{3}$ for $V$ were used. Figure 5 shows the curve resulting from the fitting with a $K_{\text {ad }}$ value of $45 \mathrm{~cm}$, together with the observed TIRF intensities. A good agreement between the fitting curve and the observed intensities supports the adsorption mechanism proposed here.

Further, the same type analysis was carried out for a DEHP/water system containing $1.99 \times 10^{-7} \mathrm{~mol} \mathrm{dm}^{-3}$ CTAB; the adsorption constant was determined to be $1.2 \mathrm{~cm}$ (Fig. 6). The difference in the adsorption constant between the toluene/water and DEHP/water systems may be due to a difference in the solvation of the trimethylammonium group of $\mathrm{CTA}^{+}$. Namely, toluene

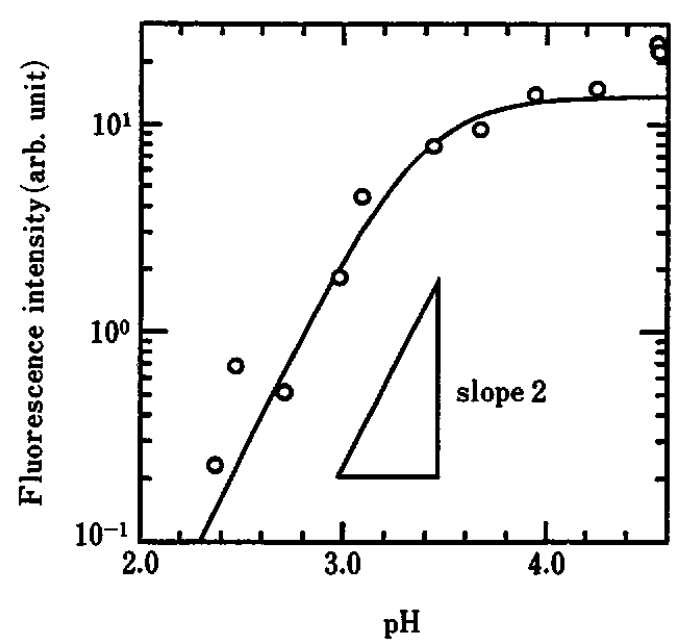

Fig. 6 Best-fitted curve resulting from a stoichiometric analysis of the TIRF intensities with an adsorption constant of $1.2 \mathrm{~cm}$ at the DEHP-water interface. The aqueous phase contained $1.98 \times 10^{-6} \mathrm{~mol} \mathrm{dm}^{-3}$ TPPS and $1.99 \times 10^{-7} \mathrm{~mol}$ $\mathrm{dm}^{-3}$ CTAB. The TIRF was measured at $654 \mathrm{~nm}$.

molecules hardly solvate the ammonium group because of their hydrophobic nature. As a result, a large fraction of the ammonium group is excluded from the toluene phase and immersed to the aqueous phase for the $\mathrm{CTA}^{+}$ions at the interface. On the other hand, because DEHP tends to solvate the ammonium group due to a relatively large dielectric constant $(\varepsilon=5.1)$, the ammonium group tends to stay in the DEHP phase. Hence, the toluene-water interface facilitates the ionassociation to a higher degree than the DEHP-water interface, leading to the larger adsorption constant.

\section{External electric field effect on the adsorption of TPPS}

To investigate an electric field effect, the DEHP-water interface was employed, because a high viscosity of DEHP ( $\eta=56.5$ poise) was available for keeping a flat interface, even while applying an electric field. The field was applied with respect to the aqueous phase. Figure 7 shows the $\mathrm{pH}$ dependencies of the TIRF intensity at three applied voltages. When the voltage was $+0.5 \mathrm{kV}$, the TIRF intensities (open circle) were nearly equal to those (solid circle) at $0 \mathrm{~V}$, whereas the application of $-0.5 \mathrm{kV}$ weakened the TIRF intensity (open square) to almost 0 . This means that the negative voltage forced the unprotonated TPPS to move away from the interfacial region; a positive voltage kept it residing in the interfacial region. This phenomenon can be explained in terms of an electrophoretic migration and the hydrophilic nature of the ionic species of TPPS. Because the electric field is formed in the direction from the aqueous phase toward the organic phase when applying a negative voltage, the negatively charged unprotonated TPPS is expected to migrate from the interfacial region toward the bulk in the aqueous phase. On the other hand, in the case of a positive voltage, although the negatively charged TPPS tends to go toward the organic phase, it cannot transfer 


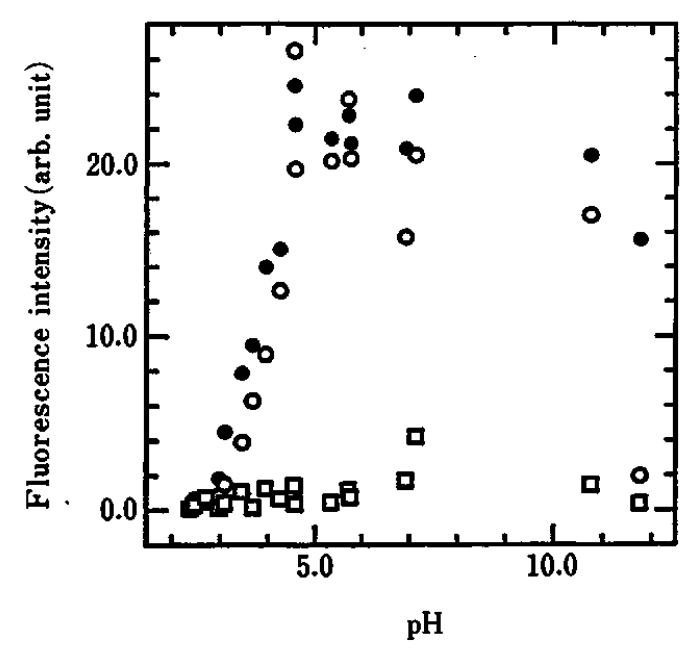

Fig. 7 Electric field effect on the ion-association adsorption at the DEHP-water interface. Solid circle, $0 \mathrm{~V}$; open circle, $+0.5 \mathrm{kV}$ for $1 \mathrm{~h}$; open square, $-0.5 \mathrm{kV}$ for $2 \mathrm{~h}$; The voltage was applied with respect to the aqueous phase. The aqueous phase contained $1.98 \times 10^{-6} \mathrm{~mol} \mathrm{dm}^{-3}$ TPPS and $1.99 \times 10^{-7} \mathrm{~mol}$ $\mathrm{dm}^{-3}$ CTAB.

into the organic solvent because of its poor solubility.

Figure 8 shows the voltage dependence of the TIRF intensity, indicating that the higher is the negative voltage, the lower is the TIRF intensity. Because we have little knowledge concerning the electric field around the organic solvent-water interface, the precise mechanism of this field effect must be determined by a further study. In any event, it was demonstrated that the ionassociation adsorption could be controlled by applying an external electric field. Control by an electrostatic technique will lead to a simple and selective separation method.

The authors thank Miss Yoriko Saitoh for her valuable preliminary experiments. This work was supported by Grantin-Aids for General Scientific Research from the Ministry of Education, Science, Sports and Culture, Japan (Nos. 06554035 and 07404042).

\section{References}

1. H. Watarai and Y. Chida, Anal. Sci., 10, 105 (1994).

2. H. Watarai and Y. Shibuya, Bull. Chem. Soc. Jpn., 62, 3446 (1989).

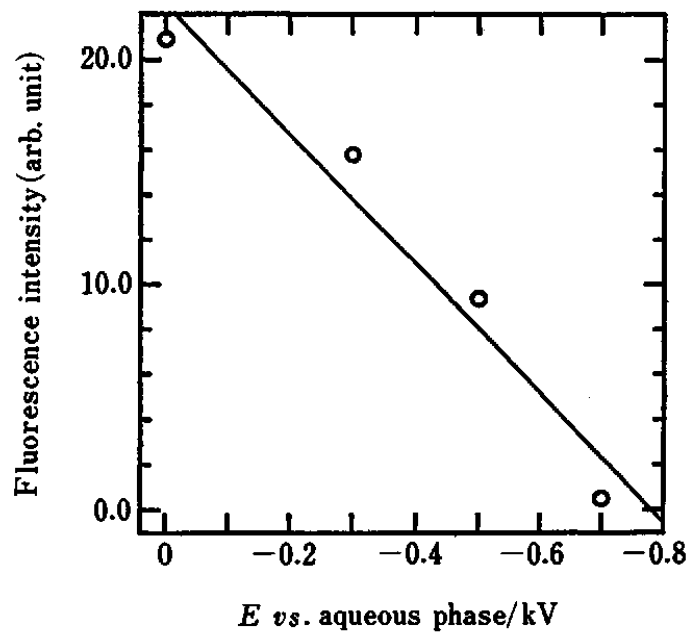

Fig. 8 Electric field dependence of the TIRF intensity at $654 \mathrm{~nm}$ in DEHP-water system. A negative voltage was applied for $2 \mathrm{~h}$ to the organic phase with respect to the aqueous phase. The $\mathrm{pH}$ was within 6.9-7.1.

3. H. Watarai and Y. Saitoh, Chem. Lett., 1995, 283.

4. F. Funaki and H. Watarai, submitted for publication.

5. P. A. Suci and W. M. Reichert, Appl. Spectrosc., 42, 120 (1988).

6. X-Z. Wu, T. Kitamori, N. Teramae and T. Sawada, Bull. Chem. Soc. Jpn., 64, 2710 (1991).

7. F. M. Mirabella Jr. and N. J. Harrick, "Internal Reflection Spectroscopy: Review and Supplement”, p. 80, Marcel Dekker, New York, 1985.

8. M. Yamaguchi, "Liquid-liquid Extraction Equipment", ed. J. C. Godfrey and M. J. Slater, Chap. 16, p. 585-624, John Wiley \& Sons, New York, 1994.

9. H. Watarai, "Solvent Extraction in the Process Industries", ed. D. H. Logsdail and M. J. Slater, Vol. 3, p. 1728 - 1732, Elsevier, London, 1993.

10. The penetration depth is defined as a distance at which an intensity of the evanescent wave falls to $1 / e$ of that at the interface. N. J. Harrick, "Internal Reflection Spectroscopy", p. 30, John Wiley \& Sons, New York, 1967.

11. J. Itoh, T. Yotsuyanagi and K. Aomura, Anal. Chim. Acta, 74, 53 (1975).

12. M. Tabata and M. Tanaka, J. Chem. Soc., Chem. Commun., 1985, 42.

13. A. Stone and E. B. Fleischer, J. Am. Chem. Soc., 90, 2735 (1968).

(Received January 11, 1996) (Accepted February 14, 1996) 